\title{
A RARE CASE OF CAMPOMELIC DYSPLASIA: A CASE REPORT
}

\author{
Kovachev E. ${ }^{1}$, B. Balev ${ }^{2}$, M. Veseli ${ }^{1}$ \\ ${ }^{1}$ Department of Obstetrics and Gynecology, Medical University, Varna; ${ }^{2}$ Department of Imaging \\ Diagnostics and Radiotherapy, Medical University, Varna
}

\section{Reviewed by: prof. I. Kozovski}

\begin{abstract}
We report an uncommon case of campomelic dysplasia in a 24-year-old patient nuliparva, pregnant in 38 g.w. with breech presentation, admitted at the Specialized Obstetrics and Gynecology Hospital. The ultrasound examination visualized Intrauterine Growth Retardation (IUGR) as well as severe angulation and rhizomelic shortening of the femur and tibia. Those ultrasonography features suggested the presence of campomelic dysplasia. After a planned Cesarian section, a female baby was delivered, weighing $2250 \mathrm{gr}$. The postnatal $\mathrm{X}$ ray was managed and the diagnosis was confirmed without other abnormallites. The genetic testing established normal karyotypes of the parents and we suggested the new SOX 9 mutation of the baby. No individual feature is pathognomonic of this condition, however the most typical characteristic sign of campomelic dysplasia is the marked anterior bowing of the long bones, particularly of the femur and tibia.
\end{abstract}

Key words: Campomelic dysplasia, campomelic dwarfism, pregnancy

\section{INTRODUCTION}

Campomelic dysplasia (CMD) was first described by Maroteaux et al in 1971 (7). Synonyms of this disorder are camptomelic dysplasia, campomelic syndrome, campomelic dwarfism, congenital bowing of the limbs.

The disorder varies between 0,05 and 1,6:10,000 live births and can be found in all ethnic groups.

CMD is a rare congenital skeletal disorder which is characterized by the development of abnormal curvature of the long bones, particularly the lower extremites, such as femur and tibiae $(1,11)$. The syndrome has a number of other skeletal and extraskeletal features $(2,3)$.

Some authors have classified the disease into two varieties: "long limbed" and "short limbed", depending on the type of limbs involved in the pathologic process (2).

Two short-bone varieties of CMD can be distinguished, representing clear syndromes:

1. The normocephalic form, known also as kyphomelic dysplasia.

2. The craniostenotic type, which is identical to Antley Bixler syndrome.

For example, campomelic dysplasia, localized to chromosome 17 is associated with XY phenotypic sex reversal. The SRY gene is specific to the Y chromosome and is expressed in the human single-cell zygote immediately after

\footnotetext{
Address for correspondence:

E. Kovachev, Dept. of Obstetrics and Gynecology,

Medical University, Varna; SBAGAL "Prof. d-r D. Stamatov",

150, Tsar Osvoboditel Blvd., 9000 Varna - Bulgaria

e-mail: kovachev_md@abv.bg
}

ovum fertilization and the SOX 9 mutation is a cause of $\mathrm{CMD}$ and gonadal or testicular dysgenesis (8).

SOX 9 is also responsible for synthesis of collagen type II, and mutations of SOX 9 result in CMD which is a cartilage and skeletal malformation syndrome with congenital angulation and bowing of long bones that is associated with 46, XY complete gonadal dysgenesis in about three fourths of the $\mathrm{XY}$ cases $(4,5)$.

Sex reversal occurs in some genotypic male fetuses who lack the H-Y antigen. Phenotypic sex ratio is approximately $\mathrm{M} 1: \mathrm{F} 2.3$, karyotipic sex ratio is approximately M2:F1.

The transmission of CMD is still debated. Autosomal-recessive inheritance is considered to be the most common pattern, although it may also be a result of sporadic autosomal-dominant mutation (1).

The recurrence risk depends on the etiology. If transmitted by autosomal-recessive pattern, there is a $25 \%$ recurrence risk. When transmitted by autosomal-dominant pattern, it has $50 \%$ recurrence risk, but in fact, most are new mutations $(1,8)$.

The most characteristic sign of CMD is the marked anterior bowing of the long bones, particularly of the femur and tibia and severe angulation may mimic fractures (4). Other sonographic features that are commonly present include growth restriction; bell-shaped narrow chest; 11 pair of ribs; hypoplasia of the midthoracic vertebral bodies, fibula, and scapula; scoliosis; shortness of the limbs; talipes equinovarus; tracheobronchomalacia and respiratory distress; flat and small face; high forehead with prominent occiput; low nasal bridge; micrognathia; macrocephaly; cleft of the soft palate; hypertelorism; low-set and mal- 
formed ears; hydrocephalus and ambiguous genitalia $(1,4,10,11)$.

A kyphosis or scoliosis was not a common finding on neonatal $\mathrm{x}$ rays, but featured more frequently in surviving cases (9).

\section{CASE PRESENTATION}

A 24-year-old primigravida with probable term of delivery 24.10.2011, without any accompanying diseases or preceding abdominal surgeries. The patient was hospitalized at 38th week of gestation due to intrauterine growth retardation of the fetus. The patient had not visited pregnancy consultation regularly and ultrasound fetal morphology had not been performed. The patient was accepted at the Specialized Obstetrics and Gynecology Hospital and the routine transabdominal ultrasound examination with linear probe (3-7 MHz Sonoace 4; Medison) proved lower limbs abnormalities. Severe angulation of the femur was visualized, in combination with reduced size (fig. 1).

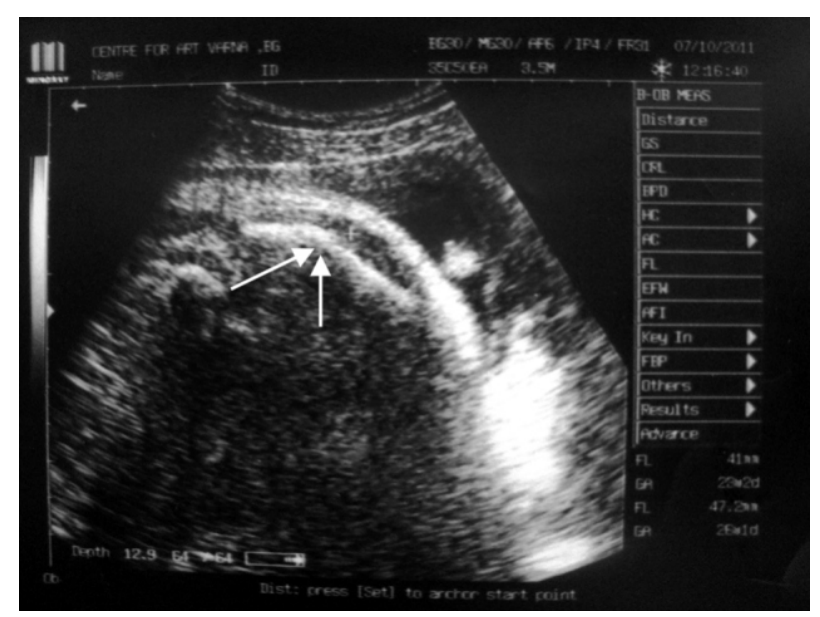

Fig. 1. Ultrasound view of the femur with severe angulation (our case).

A slight shortening of the tibial bones was discovered with deviation of mesomelic type. The bone structure of the rest of the axial skeleton was normal, with normal epiphuseal nuclei, retarded bone maturation was not observed. All biometric indicators were lower than the actual gestational age; the shortening and the angulation of the femurs were most obvious. Other abnormalities were excluded. The following illnesses were taken into consideration in differential diagnostic aspect: osteogenesis imperfecta, short-limb dysplasias, thanatophoric dysplasia, hypophosphatasia, arthrogryposis. Due to the observed pathological changes of the femur and the lack of changes of other bone structures, we were directed to the diagnosis CMD. The patient was hospitalized and Cesarian section planned, due to breech presentation, primigravida, IUGR, unfavourable obstetric factors. The surgery was performed without any complication, a living female baby was extracted in breech presentation, weighing $2250 \mathrm{gr}$ and $46 \mathrm{~cm}$ long, Apgar score 8 at 1 minute, 9 at 5 minutes (fig. 3). The examination

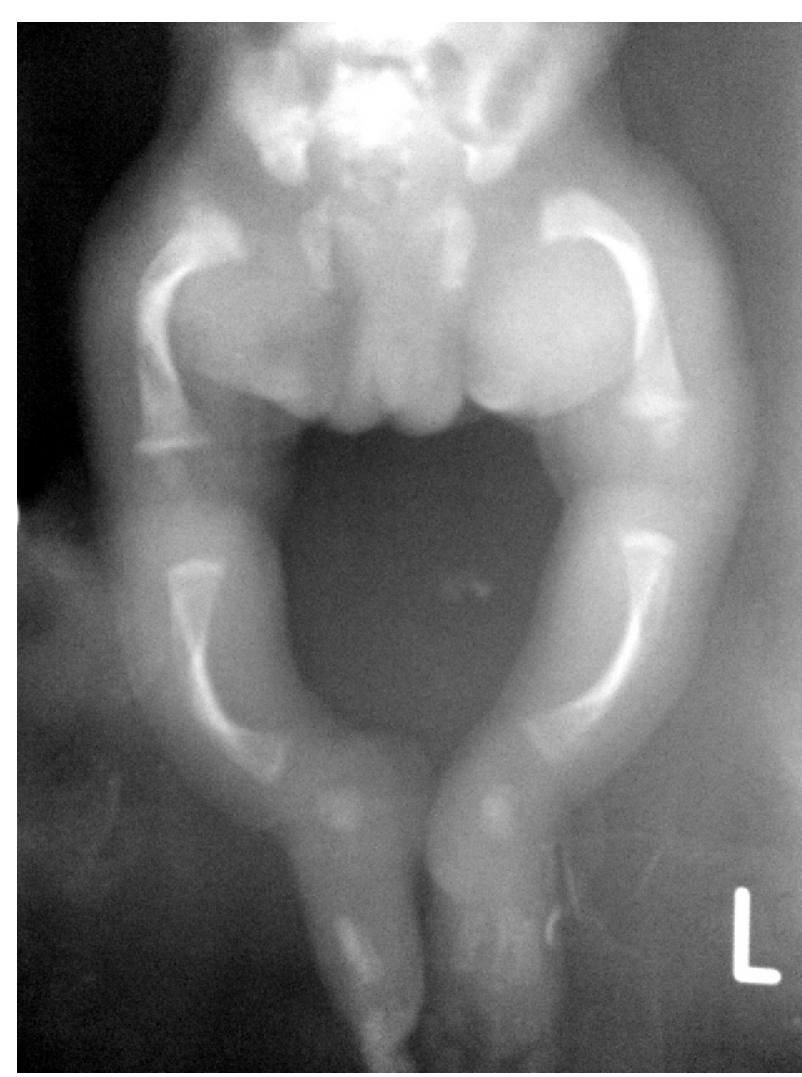

Fig. 2. Postnatal $x$-ray visualization of the femurs and the tibiae bowing (our case).

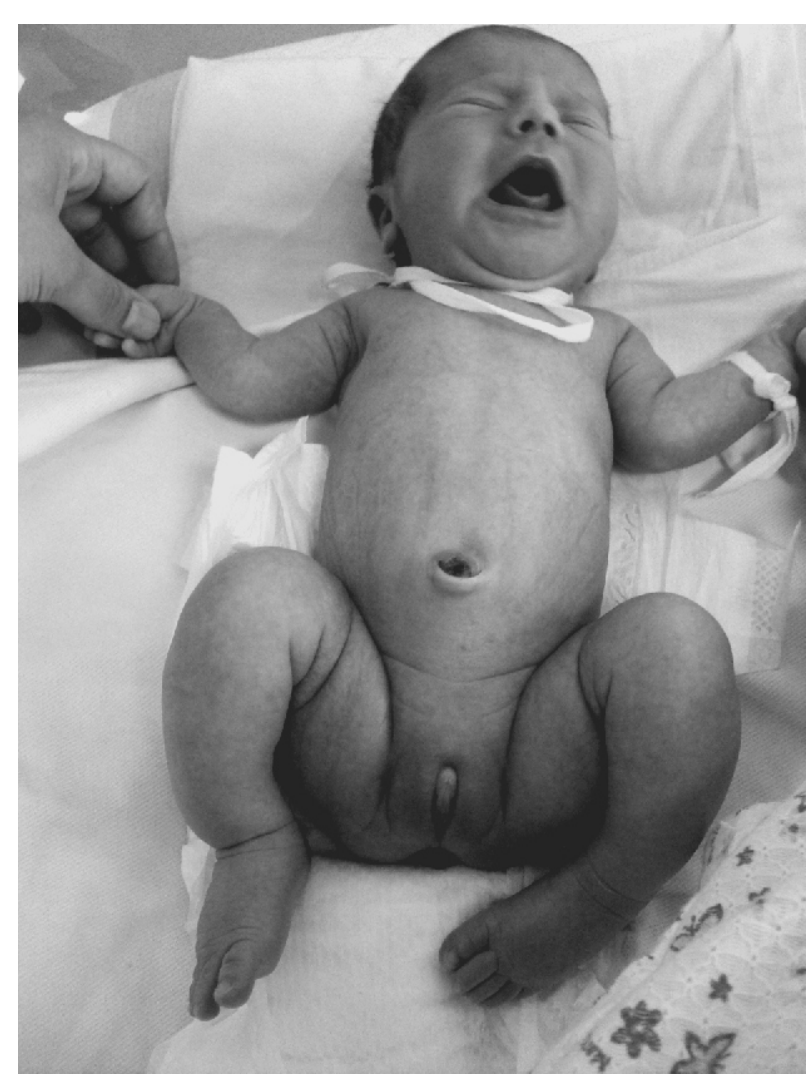

Figure 3. Image of the baby with hips shortening and lower thighs deviation (our case). 
of the baby showed shortening with deviation of the hips and the lower thighs (clubfoot). Postnatal x-ray examination of the whole body was performed, proving symmetrical shortening and deviation of the lower tibular bones of rhizomelic and mesomelic types (fig. 2).

The pediatric orthopedic examination confirmed the diagnosis and recommended x-ray check-ups of the skeleton every 3 months and a possible surgery with splinting of the extremities if necessary.

The genetic testing of the parents established normal karyotypes, autosomal domination and autosomal recessive inheritance was not discovered. The chromosome constellation of the infant was $46 \mathrm{XX}$, and new SOX 9 mutation was suggested.

\section{DISCUSSION}

Campomelic dysplasia is a rare skeletal disorder that is usually lethal. It is characterized by bowing of the lower limbs, severe respiratory distress, and many of the chromosomal (XY) males show sex reversal. Patients with a chromosomal rearrangement involving $17 \mathrm{q}$ show a milder phenotype. An unusual aspect of CMD is that some patients with male karyotypes have female or ambiguous genitalia with a spectrum of sexual differentiation. The histology of the ovaries in sex reversed males varies from gonads with testicular differentiation to dysgenetic gonads with primary follicles and in some cases, they should be surgically removed because an increased chance for malignancy exists.

The diagnosis of CMD can be confirmed through genetic testing, which requires blood sample from the affected individual and identifying the specific alteration in the SOX 9 gene (8).

The diagnosis can be strongly suspected when femur and tibial bowing is noted and the diagnosis can be done with amniocentesis.

CMD is associated with a significant risk for death in the newborn period due to small chest, small lungs and tracheomalacia (6).
An orthopedist should also be consulted regarding the other bone problems such as clubfoot and bowed legs.

No individual feature is pathognomonic of this condition, but no other syndrome has this combination of features.

\section{REFERENCES}

1. Callen, Peter W. Ultrasonography in Obstetrics and Gynecology. Fift Edition, Saunders Elsevier, 2008; 127:128.

2. Cordone M, Lituania M, Zampatti C, et al: In utero ultrasonography features of campomelic dysplasia. Prenat Diagn, 1989; 9:745.

3. Cunningham G. Williams Obstetrics, Twenty-Third Edition, The McGraw-Hill Companies, Inc, 2010; 101.

4. Huang B, et al: Autosomal XX sex reversal caused by duplication of SOX 9, Am J Med Genet 1999; 87:349.

5. Khajavi A, Lachman R, Rimoin N, et al: Heterogeneity in the campomelic syndromes. Long and short bone varieties. Radiology, 1976; 120:641.

6. Lee FA, Isaacs H, Strauss J: The "campomelic" syndrome. Am J Dis Chi, 1972; 124:485.

7. Maroteaux P, Spranger J, Opitz JM, et al. Le syndrome campomelique. Presse Med 1971; 79: 1157-62.

8. Martin R, Fanaroff A, Walsh M. Fanaroff and Martin's Neonatal-Perinatal Medicine. Diseases of the fetus and infant. Vol. 2; $9^{\text {th }}$ Edition. Elsevier Mosby; 2011; 1602:1603.

9. Normann EK, Pedersen JC, Stiris G, et al. Campomelic dysplasia - an underdiagnosed condition? Eur J Pediatr 1993; 152:331-3.

10. Shumacher R, Seaver L, Spranger U. Fetal Radiology: A Diagnostic Atlas. Second Edition, Springer-Verlag Berlin Heidelberg, 2010; 124.

11. Ward JC, Gross SJ, Tharapel AT, et al. Report of campomelic dysplasia in paternal half-siblings without apparent consanguinity or parental expression. Am J Hum Genet 1994; 3(suppl 55):538A. 